\title{
Renormalizability of Supersymmetric Group Field Cosmology
}

\author{
Sudhaker Upadhyay* \\ S. N. Bose National Centre for Basic Sciences, \\ Block JD, Sector III, Salt Lake, Kolkata -700098, India.
}

\begin{abstract}
In this paper we consider the gauge invariant third quantized model of supersymmetric group field cosmology. The supersymmetric BRST invariance for such theory in non-linear gauge is also analysed. The path integral formulation to the case of a multiverse made up of homogeneous and isotropic spacetimes filled with a perfect fluid is presented. The renormalizability for the scattering of universes in multiverse are established with suitably constructed master equations for connected diagrams and proper vertices. The Slavnov-Taylor identities for this theory hold to all orders of radiative corrections.
\end{abstract}

\section{INTRODUCTION}

In nonperturbative quantization of gravitational degrees of freedom the background-independent loop quantum gravity is widely investigated [1 3$]$. In this quantization scheme the constraints are written in terms of the densitized triad and of the Ashtekar-Barbero connection [4 9]. The main difficulties of loop quantum gravity quantization scheme are the lack of complete definition of the quantum dynamics and the proof that leads back the resulting theory to Einsteins gravity. The complete definition of the quantum dynamics of spin network states has been obtained by embedding loop quantum gravity states into the larger framework of group field theories [10, 11] via spin-foam models [12, 13]. These are basically quantum field theories on group manifolds and the Feynman amplitudes of group field theories are spinfoam models. The loop quantum gravity is a second quantized object with fixed topology. However, the topology changing processes can not be analysed through second quantization approach and therefore we need the third quantization to analyse such processes [14 17]. The basic idea of the third quantization formalism is to treat the many-universe system as a quantum field theory on superspace [18]. The name third quantization comes from the fact that the field which is quantized is the wave function of the universe, and it depends on the particles existing within the universe. The third quantization of loop quantum gravity naturally leads to the group field theory [19 22]. The minisuperspace (Wheeler-De Witt) approximations to group field theory are known as group field cosmology [23 30].

Furthermore, the supersymmetry has been proved as an important ingredient in the study of Mtheory [31 33] which provides a basis for many phenomenological models beyond the standard model [34, 35]. The supersymmetry is also a prominent candidate for dark matter [36]. A supersymmetric generalization of group field cosmology, called as super-group field cosmology, has been studied very recently as a model for multiverse [37]. However, a variety of multiverse hypotheses have been considered from different cosmological viewpoints [38]. The super-group field cosmology remains invariant under gauge symmetry transformation and therefore contains some unphysical degrees of freedom. The spurious degree of freedom can be removed by choosing a suitable gauge-fixing condition and this can be achieved by adding a term, called as gauge-fixing term, in the action at quantum level. This gauge-fixing term reflects the Faddeev-Popov ghosts in the void (the vacuum state of the multiverse) functional (corresponds to vacuum functional in the second quantization) to complete the effective theory. The supersymmetric BRST transformation as well as unitarity for the theory of multiverse has discussed very recently [39]. So, it is worthwhile to explore the identities between all connected and disconnected Green functions to proof the algebraic renormalizability of multi-universe. This is the motivation of our present investigation.

In this paper we analyse the gauge invariance of super-group field cosmology which is a model for ho-

*Electronic address: sudhakerupadhyay@gmail.com; sudhaker@boson.bose.res.in 
mogeneous and isotropic multiverse filled with a perfect fluid. The linear and non-linear BRST invariance for such model of mutiverse are written explicitly. The Jacobian for such BRST transformation is unit. The symmetry generator annihilates the physical states of total Hilbert space to prove the unitarity of the scattering matrix. We construct the void functional for third quantized cosmological model in terms of supersources. The BRST invariance of the generating functional leads to the master equations for connected Green's functions and for proper vertices. The renormalizability of the theory is proved by checking the consistency condition of the the Salvnov-Taylor identities. Here we notice that the Jacobian for path integral measure under BRST transformation is unit.

The plan of the paper is as follows. In section II, we discuss the supersymmetric group cosmology. The analyses of the third quantized BRST transformations for super-group field cosmology are made in section III. Section IV is devoted to establish the renormalizability for the theory of multiverse. The last section is reserved for discussions and conclusions.

\section{SUPERSYMMETRIC GROUP FIELD COSMOLOGY}

Let us consider a quantum multiverse made up of homogeneous and isotropic universes filled up with a perfect fluid. Now, we start with the loop quantum cosmology with a massless scalar field $\phi$ as matter in a spatially flat, homogeneous and isotropic universe. The four-dimensional metric is then given by

$$
d s^{2}=-N^{2}(t) d t^{2}+a^{2}(t) \delta_{a b} d x^{a} d x^{b},
$$

where $N(t)$ and $a(t)$ are lapse function and scaler factor respectively. Here the spatial indices are labelled by Latin indices $a, b, \ldots=1,2,3$. The variables used in loop quantum gravity are the densitized triad $E_{i}^{a}$ and the Ashtekar-Barbero connection $A_{a}^{i}=\gamma\left(\omega_{0}^{i}\right)_{a}$, where $\gamma$ is the Barbero-Immirzi parameter and $\left(\omega_{0}^{i}\right)_{a}$ is the spin connection compatible with the triad. The curvature of $A_{a}^{i}$ in the loop quantum cosmology is expressed through the holonomy around a loop such that the area of a loop cannot be smaller than a fixed minimum area because the smallest eigenvalue of the area operator in loop quantum gravity is non-zero. Now one defines the eigenstates of the volume operator $\mathcal{V}$ with a basis, $|\nu\rangle$, are $\mathcal{V}|\nu\rangle=2 \pi \gamma G|\nu||\nu\rangle$, where gravitational conguration variable $\nu= \pm a^{2} \mathcal{V}_{0} / 2 \pi \gamma G$ has the dimensions of length. The Hamiltonian constraint for a homogeneous isotropic universe (in the Plank units) is defined as 28]

$$
-B(\nu)\left[E^{2}-\partial_{\phi}^{2}\right] \Phi(\nu, \phi)=K^{2} \Phi(\nu, \phi)=0,
$$

where $\Phi(\nu, \phi)$ is a wave function on configuration space and $E^{2}$ is a difference operator of the form:

$$
-B(\nu) E^{2} \Phi(\nu, \phi)=C^{+}(\nu) \Phi\left(\nu+\nu_{0}, \phi\right)+C^{0}(\nu) \Phi(\nu, \phi)+C^{-}(\nu) \Phi\left(\nu-\nu_{0}, \phi\right),
$$

and $K_{\mu}$ is defined as $K_{\mu}=\eta_{\mu \nu} K^{\nu}=\left(\sqrt{B(\nu)} \partial_{\phi}, \sqrt{B(\nu)} E\right)$ with metric $\eta_{\mu \nu}=\operatorname{diag}(+1,-1)$. So, $K^{2}=$ $K_{\mu} K^{\mu}=-B(\nu)\left[E^{2}-\partial_{\phi}^{2}\right]$. Here $\nu_{0}$ is an elementary length unit, usually defined by the square root of the area gap and the functions $B(\nu), C^{+}(\nu), C^{0}(\nu)$ and $C^{-}(\nu)$ depend on the choice of the lapse function and on the details of quantization scheme. For particular choice of lapse function, $N=1$, in an improved dynamic scheme, these functions have following form [40]:

$$
\begin{aligned}
B(\nu) & =\frac{3 \sqrt{2}}{8 \sqrt{\sqrt{3} \pi \gamma G}}|\nu||| \nu+\left.\frac{\nu_{0}}{4}\right|^{\frac{1}{3}}-\left.\left|\nu-\frac{\nu_{0}}{4}\right|^{\frac{1}{3}}\right|^{3}, \\
C^{+}(\nu) & =\frac{1}{12 \gamma \sqrt{2 \sqrt{3}}}\left|\nu+\frac{\nu_{0}}{2}\right||| \nu+\frac{\nu_{0}}{4}|-| \nu+\frac{3 \nu_{0}}{4}||, \\
C^{0}(\nu) & =-C^{+}(\nu)-C^{+}\left(\nu-\nu_{0}\right), \\
C^{-}(\nu) & =C^{+}\left(\nu-\nu_{0}\right) .
\end{aligned}
$$


However, for $N=a^{3}$ where $a$ is scale factor and for orientation reversal symmetric wave function the structures of these functions are

$$
\begin{aligned}
B(\nu) & =\frac{1}{\nu}, \\
C^{+}(\nu) & =\frac{\sqrt{3}}{8 \gamma}\left(\nu+\frac{\nu_{0}}{2}\right), \\
C^{0}(\nu) & =-\frac{\sqrt{3}}{4 \gamma} \nu, \\
C^{-}(\nu) & =C^{+}\left(\nu-\nu_{0}\right),
\end{aligned}
$$

and in the semiclassical limit $\nu \gg \nu_{0}$, these expressions (5) agree with (4).

By definition, the solutions of the first quantized theories correspond to the free field solutions in the second quantized formalism, the solutions of the second quantized theory should corresponds to free field solutions in the third quantized formalism. Hence, the solution of loop quantum cosmology will now correspond to the classical field of group field cosmology. Now, free field action for bosonic distribution of universes, of which classical solution reproduces the Hamiltonian constraint for loop quantum gravity, is given by [28]

$$
S_{b}=\sum_{\nu} \int d \phi \mathcal{L}_{b}=\sum_{\nu} \int d \phi \Phi(\nu, \phi) K^{2} \Phi(\nu, \phi),
$$

where $\Phi(\nu, \phi)$ to be a real scalar field.

It is worthwhile to analyse the fermionic distribution of universes also which might lead to correct the value of the cosmological constant. Since the correct value of cosmological constant is not obtained by considering only bosonic distributions of universes in the multi-universe [41]. Consequently, free action corresponding to fermionic group field cosmology is constructed as [37]

$$
S_{f}=\sum_{\nu} \int d \phi \Psi^{b}(\nu, \phi) K_{b}^{a} \Psi_{a}(\nu, \phi)
$$

where $\Psi_{a}(\nu, \phi)=\left(\Psi_{1}(\nu, \phi), \Psi_{2}(\nu, \phi)\right)$ is a fermionic spinor field and $K_{a b}$ is defined as $K_{a b}=\left(\gamma^{\mu}\right)_{a b} K_{\mu}$. The spinor indices are raised and lowered by the second-rank antisymmetric tensors $C^{a b}$ and $C_{a b}$, respectively. These tensors satisfy following condition $C_{a b} C^{c b}=\delta_{a}^{c}[42]$. Fermionic statics will not change the dynamics of a single universe by requirement that the free action (17) will lead to the Hamiltonian constraint of loop quantum gravity. The above bosonic and the fermionic actions describe bosonic and the fermionic universes in the multiverse and hence, it is worthwhile to construct a supersymmetric gauge invariant multiverse. The main idea behind the third quantization is: to treat the many-universe system as a quantum field theory on superspace. For this purpose we define, two complex scalar super-group fields $\Omega(\nu, \phi, \theta)$ and $\Omega^{\dagger}(\nu, \phi, \theta)$ and a spinor super-group field $\Gamma_{a}(\nu, \phi, \theta)$, which are suitably contracted with generators of a Lie algebra, $\left[T_{A}, T_{B}\right]=i f_{A B}^{C} T_{C}$, as

$$
\begin{aligned}
\Omega(\nu, \phi, \theta) & =\Omega^{A}(\nu, \phi, \theta) T_{A}, \\
\Omega^{\dagger}(\nu, \phi, \theta) & =\Omega^{\dagger A}(\nu, \phi, \theta) T_{A} \\
\Gamma_{a}(\nu, \phi, \theta) & =\Gamma_{a}^{A}(\nu, \phi, \theta) T_{A} .
\end{aligned}
$$

The extra variable $\theta$ are Grassmannian in nature which defines the extra direction in superspace. The super-covariant derivative of these superfields is defined as [37]

$$
\begin{aligned}
\nabla_{a} \Omega^{A}(\nu, \phi, \theta) & =D_{a} \Omega^{A}(\nu, \phi, \theta)-i f_{C B}^{A} \Gamma_{a}^{C}(\nu, \phi, \theta) \Omega^{B}(\nu, \phi, \theta), \\
\nabla_{a} \Omega^{A \dagger}(\nu, \phi, \theta) & =D_{a} \Omega^{A \dagger}(\nu, \phi, \theta)+i f_{C B}^{A} \Omega^{C \dagger}(\nu, \phi, \theta) \Gamma_{a}^{B}(\nu, \phi, \theta),
\end{aligned}
$$

where super-derivative $D_{a}=\partial_{a}+K_{a}^{b} \theta_{b}$. We also define the field strength for a matrix valued spinor field $\left(\Gamma_{a}^{A}\right)$ as follows $\omega_{a}^{A}(\nu, \phi, \theta)=\nabla^{b} \nabla_{a} \Gamma_{b}^{A}(\nu, \phi, \theta)$. With these introduction, now, we are able to write the 
the classical action for the super-group field cosmology as (for details see e.g. [37])

$$
S_{0}=\sum_{\nu} \int d \phi\left[D^{2}\left\{\Omega_{A}^{\dagger}(\nu, \phi, \theta) \nabla^{2} \Omega^{A}(\nu, \phi, \theta)+\omega_{A}^{a}(\nu, \phi, \theta) \omega_{a}^{A}(\nu, \phi, \theta)\right\}\right]_{\text {, }}
$$

where, denotes $\theta=0$ after performing calculations. This supersymmetric action remains invariant under following gauge transformations

$$
\begin{aligned}
\delta \Omega^{A}(\nu, \phi, \theta) & =i f_{C B}^{A} \Lambda^{C}(\nu, \phi, \theta) \Omega^{B}(\nu, \phi, \theta), \\
\delta \Omega^{A \dagger}(\nu, \phi, \theta) & =-i f_{C B}^{A} \Omega^{C \dagger}(\nu, \phi, \theta) \Lambda^{B}(\nu, \phi, \theta), \\
\delta \Gamma_{a}^{A}(\nu, \phi, \theta) & =\nabla_{a} \Lambda^{A}(\nu, \phi, \theta) .
\end{aligned}
$$

where $\Lambda^{A}$ is an infinitesimal local parameter. The gauge symmetry reflects that theory posses some redundant degrees of freedom. To quantize theory correctly we need to remove them. In next section, we will show how these can be achieved for this theory.

\section{THE BRST SYMMETRIES AND THE PHYSICAL STATES}

In this section we discuss the nilpotent symmetries for the theory in linear [39] and in non-linear gauges. For this purpose, our first goal is to remove the gauge redundancy by fixing a gauge. Making analogy with ordinary supersymmetric gauge theory, we chose the following covariant gauge condition:

$$
D^{a} \Gamma_{a}^{A}(\nu, \phi, \theta)=0 .
$$

This can be incorporated at a quantum level by adding the appropriate gauge-fixing term to classical action which breaks the gauge symmetry. The linearised gauge-fixing term in Landau gauge using Nakanishi-Lautrup auxiliary superfield $B^{A}(\nu, \phi, \theta)$ is given by

$$
S_{g f}=\sum_{\nu} \int d \phi\left[D^{2}\left\{B_{A}(\nu, \phi, \theta) D^{a} \Gamma_{a}^{A}(\nu, \phi, \theta)\right\}\right]_{\mid} .
$$

The effect of the gauge-fixing term in the exponent of path integral can be compensated by additional Faddeev-Popov ghost term. In this case the ghost term is constructed as

$$
S_{g h}=\sum_{\nu} \int d \phi\left[D^{2}\left\{\bar{c}_{A}(\nu, \phi, \theta) D^{a} \nabla_{a} c^{A}(\nu, \phi, \theta)\right\}\right]_{\mid},
$$

where $c^{A}(\nu, \phi, \theta)$ and $\bar{c}^{A}(\nu, \phi, \theta)$ are the ghost and anti-ghost superfields respectively. Now the total action

$$
S_{T}=\sum_{\nu} \int d \phi \mathcal{L}_{T}=S_{0}+S_{g h}+S_{g f}
$$

remains invariant under following third quantized BRST transformations

$$
\begin{aligned}
s \Omega^{A}(\nu, \phi, \theta) & =i f_{C B}^{A} c^{C}(\nu, \phi, \theta) \Omega^{B}(\nu, \phi, \theta), \\
s \Omega^{A \dagger}(\nu, \phi, \theta) & =-i f_{C B}^{A} \Omega^{\dagger C}(\nu, \phi, \theta) c^{B}(\nu, \phi, \theta), \\
s c^{A}(\nu, \phi, \theta) & =f_{C B}^{A} c^{C}(\nu, \phi, \theta) c^{B}(\nu, \phi, \theta), \\
s \Gamma_{a}^{A}(\nu, \phi, \theta) & =\nabla_{a} c^{A}(\nu, \phi, \theta), \\
s \bar{c}^{A}(\nu, \phi, \theta) & =B^{A}(\nu, \phi, \theta), \\
s B^{A}(\nu, \phi, \theta) & =0 .
\end{aligned}
$$


It is easy to check that this transformation is nilpotent in nature, $s^{2}=0$, and therefore we are able to write the gauge-fixing and ghost terms collectively in terms of BRST variation of gauge-fixed fermion as 39]

$$
S_{g f}+S_{g h}=\sum_{\nu} \int d \phi s\left[D^{2}\left\{\bar{c}_{A}(\nu, \phi, \theta) D^{a} \Gamma_{a}^{A}(\nu, \phi, \theta)\right\}\right]_{\mid}
$$

The conserved charge corresponding to the BRST transformation using Noether's theorem is calculated as [39]

$$
\begin{aligned}
Q_{b}= & \sum_{\nu}\left[\frac{\partial \mathcal{L}_{T}}{\partial \partial_{\phi} \Gamma_{a}^{A}(\nu, \phi, \theta)} \nabla_{a} c^{A}(\nu, \phi, \theta)+\frac{\partial \mathcal{L}_{T}}{\partial \partial_{\phi} c^{A}(\nu, \phi, \theta)} f_{C B}^{A} c^{C}(\nu, \phi, \theta) c^{B}(\nu, \phi, \theta)\right. \\
& +\frac{\partial \mathcal{L}_{T}}{\partial \partial_{\phi} \bar{c}^{A}(\nu, \phi, \theta)} B^{A}(\nu, \phi, \theta)+i \frac{\partial \mathcal{L}_{T}}{\partial \partial_{\phi} \Omega^{A}(\nu, \phi, \theta)} f_{C B}^{A} c^{C}(\nu, \phi, \theta) \Omega^{B}(\nu, \phi, \theta) \\
& \left.-i \frac{\partial \mathcal{L}_{T}}{\partial \partial_{\phi} \Omega^{\dagger A}(\nu, \phi, \theta)} f_{C B}^{A} \Omega^{\dagger C}(\nu, \phi, \theta) c^{B}(\nu, \phi, \theta)\right] .
\end{aligned}
$$

The total vector superspace of the complete theory (Eq. (15)) contains various unphysical states as well as states with negative norm in addition to the physical states. Consequently, the metric of this superspace and the inner product become indefinite and a probabilistic description of the quantum theory is lost unless we can restrict to a suitable super-subspace $(|\Psi\rangle)$ with a positive definite inner product as

$$
Q_{b}|\Psi\rangle=0 .
$$

Furthermore, the $\mathcal{S}$-matrix, which is BRST invariant, enable us to write

$$
\left[Q_{b}, \mathcal{S}\right]=0 .
$$

If we define an operator $\mathcal{S}_{\text {phy }}$ which acts and correspond to the $\mathcal{S}$-matrix in the physical super-subspace of states of the theory. Then it must be unitary, i.e.

$$
\mathcal{S}_{p h y}^{\dagger} \mathcal{S}_{p h y}=\mathcal{S}_{p h y} \mathcal{S}_{\text {phy }}^{\dagger}=1
$$

The BRST invariance of the theory automatically leads to a formal proof of unitarity of the super-Smatrix in the super-subspace of the truly physical states of the theory.

Equation (19) gives us liberty to chose different gauge-fixing condition for the theory as physical states do not depend on the choice of the gauge-fixing condition. The non-linear gauge conditions play important character in second quantized field theories. The investigation of non-linear gauge condition in third quantized model of multiverse might play significant role. For this purpose, we construct the gauge-fixing and ghost terms in non-linear gauge condition for the theory of multiverse as follows:

$$
\begin{aligned}
S_{g f}+S_{g h} & =\sum_{\nu} \int d \phi\left[D ^ { 2 } \left\{B_{A}(\nu, \phi, \theta) D^{a} \Gamma_{a}^{A}(\nu, \phi, \theta)+\frac{1}{2} \bar{c}_{A}(\nu, \phi, \theta) D^{a} \nabla_{a} c^{A}(\nu, \phi, \theta)\right.\right. \\
& \left.\left.+\frac{1}{8} f_{B C}^{A} f_{A}^{G H} \bar{c}^{B}(\nu, \phi, \theta) c^{C}(\nu, \phi, \theta) \bar{c}_{G}(\nu, \phi, \theta) c_{H}(\nu, \phi, \theta)\right\}\right]_{\mid}
\end{aligned}
$$

The third quantize non-linear BRST transformations, under which the above expression (22) is invariant, 
are calculated as

$$
\begin{aligned}
s \Omega^{A}(\nu, \phi, \theta) & =i f_{C B}^{A} c^{C}(\nu, \phi, \theta) \Omega^{B}(\nu, \phi, \theta), \\
s \Omega^{A \dagger}(\nu, \phi, \theta) & =-i f_{C B}^{A} \Omega^{\dagger C}(\nu, \phi, \theta) c^{B}(\nu, \phi, \theta), \\
s c^{A}(\nu, \phi, \theta) & =f_{C B}^{A} c^{C}(\nu, \phi, \theta) c^{B}(\nu, \phi, \theta), \\
s \Gamma_{a}^{A}(\nu, \phi, \theta) & =\nabla_{a} c^{A}(\nu, \phi, \theta), \\
s \bar{c}^{A}(\nu, \phi, \theta) & =B^{A}(\nu, \phi, \theta)-\frac{1}{2} f_{B C}^{A} \bar{c}^{B}(\nu, \phi, \theta) c^{C}(\nu, \phi, \theta), \\
s B^{A}(\nu, \phi, \theta) & =-\frac{1}{2} f_{B C}^{A} c^{B}(\nu, \phi, \theta) B^{C}(\nu, \phi, \theta) \\
& -\frac{1}{8} f_{B C}^{A} f_{G H}^{C} c^{B}(\nu, \phi, \theta) c^{G}(\nu, \phi, \theta) \bar{c}^{H}(\nu, \phi, \theta),
\end{aligned}
$$

which are nilpotent in nature, i.e. $s^{2}=0$.

\section{RENORMALIZABILITY OF THE MULTIVERSE}

To study the quantum effects for third quantize group field cosmology first we define the source free void functional as

$$
\langle 0 \mid 0\rangle=Z[0]=\int \mathcal{D} M e^{i S_{T}},
$$

where $\mathcal{D} M \equiv \mathcal{D} \Omega \mathcal{D} \Omega^{\dagger} \mathcal{D} \Gamma_{a} \mathcal{D} B \mathcal{D} c \mathcal{D} \bar{c}$ is the path integral measure. The above generating functional remains invariant under the infinitesimal BRST transformation given in Eqs. (16) and (23). It is easy to calculate the Jacobian for such BRST transformations which comes unit. Further to write full effective action for the group field cosmology we need to add following external supersource term in the $S_{T}$

$$
\begin{aligned}
S_{e x t} & =\sum_{\nu} \int d \phi\left[\bar{\eta}_{A}(\nu, \phi, \theta) \Omega^{A}(\nu, \phi, \theta)+\Omega_{A}^{\dagger}(\nu, \phi, \theta) \eta^{A}(\nu, \phi, \theta)+J_{A}^{a}(\nu, \phi, \theta) \Gamma_{a}^{A}(\nu, \phi, \theta)\right. \\
& +\bar{\mu}_{A}(\nu, \phi, \theta) c^{A}(\nu, \phi, \theta)+\bar{c}^{A}(\nu, \phi, \theta) \mu_{A}(\nu, \phi, \theta)+k^{A}(\nu, \phi, \theta) B_{A}(\nu, \phi, \theta) \\
& +\bar{\zeta}_{A}(\nu, \phi, \theta) s \Omega^{A}(\nu, \phi, \theta)+\zeta^{A}(\nu, \phi, \theta) s \Omega_{A}^{\dagger}(\nu, \phi, \theta)+K_{a}^{A}(\nu, \phi, \theta) s \Gamma_{A}^{a}(\nu, \phi, \theta) \\
& \left.+\xi_{A}(\nu, \phi, \theta) s c^{A}(\nu, \phi, \theta)\right]_{\mid},
\end{aligned}
$$

where each the superfields are coupled with their external supersources. We have not only introduced supersources for all the field variables in the theory, but we have also added supersources $\left(\bar{\zeta}_{A}, \zeta_{A}, K_{a}^{A}, \xi_{A}\right)$ for the composite variations under the BRST transformation.

Now, the generating functional for Green's functions, denoting all the supersources by $J$, is given by

$$
\langle 0 \mid 0\rangle^{J}=Z[J]=e^{i W[J]}=\int \mathcal{D} M e^{i S_{e f f}},
$$

where effective action is defined as $S_{\text {eff }}=S_{T}+S_{\text {ext }}$ and $W[J]$ is the as generating functional for only connected Feynman diagrams. 
The void expectation values of superfields, in the presence of sources, can now be written as

$$
\begin{aligned}
\left\langle 0\left|\Omega^{A}(\nu, \phi, \theta)\right| 0\right\rangle^{J} & =\frac{\delta W[J]}{\delta \bar{\eta}_{A}(\nu, \phi, \theta)}, \quad\left\langle 0\left|\Omega^{\dagger A}(\nu, \phi, \theta)\right| 0\right\rangle^{J}=\frac{\overleftarrow{\delta} W[J]}{\delta \eta_{A}(\nu, \phi, \theta)} \\
\left\langle 0\left|\Gamma_{a}^{A}(\nu, \phi, \theta)\right| 0\right\rangle^{J} & =\frac{\delta W[J]}{\delta J_{A}^{a}(\nu, \phi, \theta)}, \quad\left\langle 0\left|c^{A}(\nu, \phi, \theta)\right| 0\right\rangle^{J}=\frac{\delta W[J]}{\delta \bar{\mu}_{A}(\nu, \phi, \theta)}, \\
\left\langle 0\left|\bar{c}^{A}(\nu, \phi, \theta)\right| 0\right\rangle^{J} & =\frac{\overleftarrow{\delta} W[J]}{\delta \mu_{A}(\nu, \phi, \theta)}, \quad\left\langle 0\left|B^{A}(\nu, \phi, \theta)\right| 0\right\rangle^{J}=\frac{\delta W[J]}{\delta k_{A}(\nu, \phi, \theta)} \\
\left\langle 0\left|s \Omega^{A}(\nu, \phi, \theta)\right| 0\right\rangle^{J} & =\frac{\delta W[J]}{\delta \bar{\zeta}_{A}(\nu, \phi, \theta)}, \quad\left\langle 0\left|s \Omega^{\dagger A}(\nu, \phi, \theta)\right| 0\right\rangle^{J}=\frac{\delta W[J]}{\delta \zeta_{A}(\nu, \phi, \theta)} \\
\left\langle 0\left|s \Gamma_{a}^{A}(\nu, \phi, \theta)\right| 0\right\rangle^{J} & =\frac{\delta W[J]}{\delta K_{A}^{a}(\nu, \phi, \theta)}, \quad\left\langle 0\left|s c^{A}(\nu, \phi, \theta)\right| 0\right\rangle^{J}=\frac{\delta W[J]}{\delta \xi_{A}(\nu, \phi, \theta)}
\end{aligned}
$$

The invariance of generating functional for third quantized super-group field cosmology given in Eq. (26) under BRST transformation leads to

$$
\begin{aligned}
& \sum_{\nu} \int d \phi\left[\bar{\eta}_{A}(\nu, \phi, \theta) \frac{\delta W[J]}{\delta \bar{\zeta}_{A}(\nu, \phi, \theta)}-\eta_{A}(\nu, \phi, \theta) \frac{\delta W[J]}{\delta \zeta_{A}(\nu, \phi, \theta)}+J_{A}^{a}(\nu, \phi, \theta) \frac{\delta W[J]}{\delta J_{A}^{a}(\nu, \phi, \theta)}\right. \\
& \left.+\bar{\mu}_{A}(\nu, \phi, \theta) \frac{\delta W[J]}{\delta \xi_{A}(\nu, \phi, \theta)}+\mu_{A}(\nu, \phi, \theta) \frac{\delta W[J]}{\delta k_{A}(\nu, \phi, \theta)}\right]_{\mid}=0 .
\end{aligned}
$$

This is the master equation from which we can derive all the identities relating the connected Greens functions of the multiverse by taking functional derivatives with respect to supersources.

Further, we construct the classical generating functional for proper (one particle irreducible) vertices, so-called vertex functional, using Legendre transformation as follows

$$
\begin{aligned}
\Gamma^{(0)}[\Phi, J]=W[J] & -\sum_{\nu} \int d \phi\left[\bar{\eta}_{A}(\nu, \phi, \theta) \Omega^{A}(\nu, \phi, \theta)+\Omega_{A}^{\dagger}(\nu, \phi, \theta) \eta^{A}(\nu, \phi, \theta)\right. \\
& +J_{A}^{a}(\nu, \phi, \theta) \Gamma_{a}^{A}(\nu, \phi, \theta)+\bar{\mu}_{A}(\nu, \phi, \theta) c^{A}(\nu, \phi, \theta) \\
& \left.+\bar{c}^{A}(\nu, \phi, \theta) \mu_{A}(\nu, \phi, \theta)+k^{A}(\nu, \phi, \theta) B_{A}(\nu, \phi, \theta)\right]_{\mid}
\end{aligned}
$$

where $\Phi$ and $J$ are the generic notation for superfields and supersources respectively.

Now, the master equation (Slavnov-Taylor identity) for proper vertices of Feynman diagram of universes has the following form:

$$
\begin{aligned}
\mathcal{S}\left(\Gamma^{(0)}\right)=\sum_{\nu} \int d \phi & {\left[\frac{\overleftarrow{\delta} \Gamma^{(0)}[\Phi, J]}{\delta \Omega^{A}(\nu, \phi, \theta)} \frac{\delta \Gamma^{(0)}[\Phi, J]}{\delta \bar{\zeta}_{A}(\nu, \phi, \theta)}+\frac{\delta \Gamma^{(0)}[\Phi, J]}{\delta \zeta_{A}(\nu, \phi, \theta)} \frac{\delta \Gamma^{(0)}[\Phi, J]}{\delta \Omega^{\dagger A}(\nu, \phi, \theta)}\right.} \\
& +\frac{\delta \Gamma^{(0)}[\Phi, J]}{\delta \Gamma_{a}^{A}(\nu, \phi, \theta)} \frac{\delta \Gamma^{(0)}[\Phi, J]}{\delta J_{A}^{a}(\nu, \phi, \theta)}+\frac{\overleftarrow{\delta} \Gamma^{(0)}[\Phi, J]}{\delta c^{A}(\nu, \phi, \theta)} \frac{\delta \Gamma^{(0)}[\Phi, J]}{\delta \xi_{A}(\nu, \phi, \theta)} \\
& \left.+B_{A}(\nu, \phi, \theta) \frac{\delta \Gamma^{(0)}[\Phi, J]}{\delta \bar{c}^{A}(\nu, \phi, \theta)}\right]_{\mid}=0
\end{aligned}
$$

This relationship is essential in proving the renormalizability of the super-gauge group theories. Consequently, there is no direct obstruction in calculating the Feynman diagram for multi-scattering precesses. This provides positive signature to calculate renormalizable scattering of universes in the multiverse. 


\section{THE CONSISTENCY CHECK}

To extend the Slavnov-Taylor identity to all orders of perturbation theory we consider the vertex functional written as a power series in $\hbar$

$$
\Gamma[\Phi, J]=\sum_{n=0}^{\infty} \hbar^{n} \Gamma^{(n)}[\Phi, J]
$$

The Slavnov-Taylor identity for the full vertex functional defined as

$$
\mathcal{S}(\Gamma)=0
$$

Similar to tree level approximation, we define the theory by gauge-fixing condition as,

$$
\frac{\delta \Gamma[\Phi, J]}{\delta B^{A}}=D^{2} D^{a} \Gamma_{a}^{A}
$$

To show the stability of the above expression to all order of radiative corrections, we assume that the relation (33) is valid upto $(n-1)^{t h}$ order in $\hbar$. We write the most general breaking term compatible with power-counting as

$$
\frac{\delta \Gamma[\Phi, J]}{\delta B^{A}}=D^{2} D^{a} \Gamma_{a}^{A}+\hbar^{n} \Delta^{A}+O\left(\hbar^{n+1}\right)
$$

with the breaking

$$
\Delta^{A}(\nu, \phi, \theta)=D^{2} H^{A}\left(\Gamma_{a}^{A}, \bar{c}^{A}, c^{A}\right),
$$

where $H^{A}$ is some local polynomial in $\Gamma_{a}^{A}, \bar{c}^{A}, c^{A}$. In this case the $\Delta$ satisfies following consistency condition

$$
\frac{\delta}{\delta B^{A}(\nu, \phi, \theta)} \Delta^{B}(\nu, \varphi, \theta)-\frac{\delta}{\delta B^{B}(\nu, \varphi, \theta)} \Delta^{A}(\nu, \phi, \theta)=0 .
$$

Following from $\left[\delta / \delta B^{A}(\nu, \phi, \theta), \delta / \delta B^{B}(\nu, \varphi, \theta)\right]=0$, the above condition (which is an integrability condition) shows that the breaking can be written as a functional derivative of $B^{A}(\nu, \phi, \theta)$ as

$$
\Delta^{A}(\nu, \phi, \theta)=\frac{\delta}{\delta B^{A}(\nu, \phi, \theta)} \tilde{\Delta}, \text { with } \tilde{\Delta}=\sum_{\nu} \int d \varphi D^{2}\left(B_{A} H^{A}\left(\Gamma_{a}^{A}, \bar{c}^{A}, c^{A}\right)\right)(\nu, \varphi, \theta) .
$$

The absorption of $-\tilde{\Delta}$ as a counter term at the order $n$ assures the validity of Eq. (33) up to order $n$. This shows that the gauge condition is consistent to all orders of perturbative super-group field cosmology which ends the recursive proof of renormalizability of the theory. One can also check the consistency with anti-ghost equation which must hold to all order of perturbation.

\section{CONCLUSION}

In this paper, we have considered the theory of super-group field cosmology which is a model for multiverse. In multiverse scenario, it is considered that the universes can collide with each other to form the other universes. In this consideration, the big bang and creation of the universe is a nothing more than the collision between multiverse. It is also possible that even our own universe formed because of the collision of two previous universes. It has been found that the third quantized model for multiverse is gauge invariant. Further, the third quantized linear and non-linear BRST transformations and corresponding generators have been investigated. The unitarity of scattering matrix for the physical 
processes in multiverse has been proved. We have analysed the quantum effects for super-group field cosmology through path integral approach. The requirement of renormalizabilty has been fulfilled by deriving the Slavnov-Taylor identity for the theory of cosmology. The master equations for this theory have also been derived from which all the identities relating the connected Greens functions and relations between various proper vertices can be established. The validity oft Slavnov-Taylor identity to all order of perturbation through a consistency check assures, or atleast there is no direct obstructions in, the algebraic proof of renormalizabilty of multiverse. Furthermore, we have noticed that the Jacobian for the path integral measure under BRST transformations are unit. It will be interesting to generalize the third quantized BRST transformation for this model which will lead some non-trivial Jacobian for path integral measure [43]. Since generalized BRST transformations have found many applications in the second quantized gauge field theories [44 54]. The computation of corrections in the theory of multiverse with Batalin-Vilkovisky approach will be brilliant [55].

[1] C. Rovelli, Quantum Gravity (Cambridge University Press, Cambridge, U.K., 2007).

[2] T. Thiemann, Modern Canonical Quantum General Relativity (Cambridge University Press, Cambridge, U.K., 2007)

[3] T. Thiemann, arXiv: 0110034 [gr-qc].

[4] Ashtekar, Phys. Rev. D 36, 1587 (1987).

[5] A. Ashtekar, Phys. Rev. Lett. 57, 2244 (1986).

[6] A. Ashtekar and A. Corichi, Class. Quantum Grav. 20, 4473 (2003).

[7] A. Ashtekar, Nature Phys. 2, 726 (2006).

[8] E. Livine and J. Tambornino, J. Math. Phys. 53, 012503 (2012).

[9] M. Geiller M, M. L. Rey and K. Noui Phys. Rev. D 84, 044002 (2011).

[10] D. Oriti, in Quantum Gravity, edited by B. Fauser et al. (Birkhaeuser, Basel, Switzerland, 2008) gr-qc/0512103.

[11] D. Oriti, in Foundations of Space and Time: Reflections on Quantum Gravity, edited by J. Murugan et al. (Cambridge University Press, Cambridge, U.K., 2012) arXiv:1110.5606.

[12] D. Oriti, Rep. Prog. Phys. 64, 1489 (2001).

[13] A. Perez, Class. Quantum Grav. 20, R43 (2003).

[14] A. Buonanno, M. Gasperini, M. Maggiore and C. Ungarelli, Class. Quantum Grav. 14, L97 (1997).

[15] L. O. Pimentel and C. Mora, Phys. Lett. A 280, 191 (2001).

[16] S. R. Perez and P. F. G. Diaz, Phys. Rev. D 81, 083529 (2010).

[17] V. P. Maslov and O. Yu. Shvedov, Phys. Rev. D 60, 105012 (1999).

[18] A. Strominger, Baby universes, in Quantum Cosmology and Baby Universes, Vol. 7, ed. by S. Coleman, J. B. Hartle, T. Piran and S. Weinberg, World Scientific, London (1990).

[19] A. Baratin and D. Oriti, Phys. Rev. D 85, 044003 (2012).

[20] M. Smerlak, Class. Quantum Grav. 28, 178001 (2011).

[21] A. Baratin, F. Girelli and D. Oriti, Phys. Rev. D 83, 104051 (2011).

[22] A. Tanasa, J. Phys. A: Math. Theor. 45, 165401 (2012).

[23] A. Ashtekar, J. Phys. Conf. Ser. 189, 012003 (2009).

[24] L. Qin, G. Deng and Y. Ma, Commun. Theor. Phys. 57, 326 (2012).

[25] B. Gupt and P. Singh, Phys. Rev. D85, 044011 (2012).

[26] E. W. Ewing, Class. Quant. Grav. 29, 085005 (2012).

[27] L. Qin and Y. Ma, Mod. Phys. Lett. A27, 1250078 (2012).

[28] G. Calcagni, S. Gielen and D. Oriti, Class. Quantum Grav. 29, 105005 (2012).

[29] S. Gielen, D. Oriti and L. Sindoni, Phys. Rev. Lett. 111, 031301 (2013).

[30] S. Gielen, J. Phys. Conf. Ser. 360, 012029 (2012).

[31] I. L. Buchbinder, E. A. Ivanov, O. Lechtenfeld, N. G. Pletnev, I. B. Samsonov and B. M. Zupnik, JHEP 03, 096 (2009).

[32] L. Mazzucato, Y. Oz and S. Yankielowicz, JHEP 11, 094 (2007).

[33] W. Taylor, Rev. Mod. Phys. 73, 419 (2001).

[34] M. Gozdz, W. A. Kaminski and A. Wodecki, Phys. Rev. C 69, 025501 (2004).

[35] M. Cvetic, P. Langacker and J. Wang, Phys. Rev. D 68, 046002 (2003).

[36] S. Akula, M. Liu, P. Nath and G. Peim Phys. Lett. B 709, 192 (2012). 
[37] M. Faizal, Class. Quant. Grav. 29, 215009 (2012).

[38] A. Barrau, CERN Cour. 47 N 10, 13 (2007).

[39] M. Faizal, arXiv: 1303.5477 [hep-th].

[40] A. Ashtekar, T. Pawlowski and P. Singh, Phys. Rev. D 74, 084003 (2006).

[41] S. Coleman, Nucl. Phys. B 310, 643 (1998).

[42] D. V. Belyaev and P. V. Nieuwenhuizen, JHEP 04, 008 (2008).

[43] S. Upadhyay, work in progress.

[44] S. Upadhyay and B. P. Mandal, Eur. Phys. J. C 72, 2065 (2012); Ann. Phys. 327, 2885 (2012).

[45] S. D. Joglekar and B. P. Mandal, Phys. Rev. D 51, 1919 (1995).

[46] R. Banerjee and B. P. Mandal, Phys. Lett. B 27, 488 (2000).

[47] S. Upadhyay, M. K. Dwivedi and B. P. Mandal, Int. J. Mod. Phys. A 28, 1350033 (2013).

[48] M. Faizal, B. P. Mandal and S. Upadhyay, Phys. Lett. B 721, 159 (2013).

[49] S. Upadhyay and B. P. Mandal, Eur. Phys. Lett. 93, 31001 (2011).

[50] S. Upadhyay, S. K. Rai and B. P. Mandal, J. Math. Phys. 52, 022301 (2011).

[51] S. Upadhyay and B. P. Mandal, Mod. Phys. Lett. A 40, 3347 (2010) .

[52] B. P. Mandal, S. K. Rai and S. Upadhyay, Eur. Phys. Lett. 92, 21001 (2010).

[53] S. Upadhyay, Eur. Phys. Lett. 104, 61001 (2013); Phys. Lett. B 727, 293 (2013); Ann. Phys. 340,110 (2014); Eur. Phys. Lett. 105, 21001 (2014).

[54] R. Banerjee, B. Paul and S. Upadhyay, Phys. Rev. D 88, 065019 (2013).

[55] S. Upadhyay, work in progress. 